\title{
Características sociodemográficas y motivos de consulta de las personas atendidas en el Centro de Atención Psicológica de la Universidad de Costa Rica (2004-2013)
}

\author{
Socio-demographic Characteristics and Consultation Motives of Persons \\ Attending the Psychological Services Center at the University of Costa Rica \\ (2004-2013)
}

\author{
Adriana Maroto-Vargas, Lucía Molina-Fallas \\ y Jorge Esteban Prado-Calderón \\ Universidad de Costa Rica, Costa Rica
}

\begin{abstract}
Resumen
En este estudio se tuvo como objetivo sistematizar la información sociodemográfica y los motivos de consulta de las personas atendidas en el Centro de Atención Psicológica (CAP) de la Escuela de Psicología de la Universidad de Costa Rica. Para ello, se analizaron 743 expedientes correspondientes a personas atendidas entre los años 2004 y 2013. Se encontró un aumento del 1700\% en la cantidad de casos atendidos, así como una mediana de 10 sesiones y de 56.5 días en cuanto al tiempo de espera. Poco más de la mitad de los expedientes pertenecieron a mujeres, y en su mayoría, correspondieron a personas en la adultez joven, infancia y adultez media, residentes de los cantones cercanos al CAP tales como San José, Montes de Oca, Goicoechea, Desamparados, Cartago y La Unión, $\mathrm{y}$ con diversidad de ocupaciones, aunque con preponderancia de quienes se dedicaban al estudio. Se encontró una amplia variedad de motivos de consulta que se agruparon en 20 categorías. Aunque ninguna de estas aglutinó más del 10\%, los motivos de consulta en la mayoría de los expedientes correspondieron a las categorías relacionadas con el ámbito de familia o pareja (problemas familiares, problemas de disciplina o crianza, problemas de pareja, y separación o divorcio) y a las relacionadas con el componente emocional (alteración emocional, tristeza o depresión, y agresividad). Se presentan análisis adicionales de los motivos de consulta según sexo y edad.
\end{abstract}

Palabras clave: Psicología clínica, Salud pública, Estadísticas sanitarias, Salud mental, Salud

Adriana Maroto-Vargas, Escuela de Psicología, Universidad de Costa Rica; Lucía Molina-Fallas, Coordinadora del Centro de Atención Psicológica, Escuela de Psicología, Universidad de Costa Rica; Jorge Esteban Prado-Calderón, Escuela de Psicología, Universidad de Costa Rica.

La correspondencia en relación con este artículo se dirige a Jorge Esteban Prado-Calderón, dirección electrónica: jorge.prado@ucr.ac.cr 


\begin{abstract}
This study aimed to systematize the socio-demographic information and consultation motives of those persons who attended the School of Psychology Psychological Services Center (CAP) at the University of Costa Rica. For this purpose, 743 files of patients who attended the CAP between 2004 and 2013 were analyzed. An increase of $1700 \%$ in the number of cases was found, along with a median of 10 sessions per consulting patient and 56.5 days of waiting time. Just over half of the cases were women, and most were from young adults, children and middle-aged adults, residents of cities nearby CAP such as San José, Montes de Oca, Goicoechea, Desamparados, Cartago and La Union, and with a variety of occupations, but with a preponderance of students. Furthermore, a wide variety of consultation motives was found, and they were grouped into 20 categories. Although none of these categories contains more than $10 \%$ of cases, most consultation motives correspond to categories related to the fields of the family or the couple (family problems, discipline problems or parenting, relationship problems, and break-up or divorce) and those related to the emotional component (emotional disturbance, sorrow or depression, and aggressiveness). Additional analysis of consultation motives by sex and age are presented.
\end{abstract}

Keywords: Clinical Psychology, Public Health, Health Statistics, Mental Health, Health

La Escuela de Psicología de la Universidad de Costa Rica (UCR) ha asumido el encargo de formar profesionales en Psicología desde una perspectiva de compromiso con la sociedad costarricense (Salazar, 2009). Uno de los ámbitos es la formación clínica, cuya preparación demanda al estudiantado la realización de actividades propias del quehacer profesional, tales como la atención de personas.

Desde el año 2004, existe el Centro de Proyección Comunitaria y Prácticas Estudiantiles de la Escuela de Psicología de la UCR (en adelante "Centro de Atención Psicológica" o "CAP"), el cual fue pensado como un servicio para personas cuyos recursos económicos les impiden el acceso a los servicios psicológicos en el ámbito privado y como un espacio de formación para estudiantes de la carrera de psicología (Molina, 2015).

Actualmente, el CAP ofrece un servicio de consulta psicológica (individual, pareja o familia) gratuito a personas que así lo soliciten. La atención es brindada por estudiantes de nivel avanzado de la carrera de Psicología, con la debida supervisión de una persona profesional en Psicología, incorporada al Colegio Profesional de Psicólogos de Costa Rica. La atención clínica se ofrece dentro de los periodos lectivos de la UCR y responde a las características de los cursos que se integran a trabajar en el CAP. Por lo tanto, en el primer semestre se atienden personas adultas y en el segundo semestre, personas menores de edad (Molina, 2015).

Para ofrecer un servicio de calidad clínica y que responda a los pilares de compromiso social que caracterizan a la Escuela de Psicología de la UCR, se vuelve ineludible ofrecer una estructura que contenga elementos académicos, administrativos y logísticos. Si bien cada año se presentan los informes 
correspondientes a las instancias universitarias dando cuenta del trabajo que desarrolla el CAP, es necesario ejecutar una exploración que permita tener una visión de conjunto de lo que se está desarrollando en el trabajo cotidiano, a fin de realimentarlo y fortalecerlo. Por esta razón, durante el período 2013-2015 las profesoras M.Sc. Adriana Maroto Vargas, M.Sc. Lucía Molina Fallas y el profesor Lic. Jorge Esteban Prado Calderón desarrollaron la investigación "Sistematización del perfil sociodemográfico y los motivos de consulta de las personas que han sido atendidas en el Centro de Atención Psicológica en el período 2004-2013", la cual tuvo como su principal objetivo conocer el perfil sociodemográfico y los motivos de consulta de las personas que han sido atendidas en el CAP. En el presente artículo se exponen algunos de los principales resultados de la investigación mencionada.

Al respecto, es importante indicar que en Costa Rica no existen investigaciones similares que funcionen como antecedentes del proyecto. En otros países de América Latina sí existen estudios que han reconocido la importancia de sistematizar la información sociodemográfica y los motivos de consulta de personas atendidas en centros de atención psicológica adscritos a universidades en Argentina, México y Colombia. Por ejemplo, López-Jaramillo, Montoya-Londoño y Dussán-Lubert (2012) describieron tanto las características sociodemográficas como el motivo de consulta y la impresión diagnóstica de quienes consultaron en el Centro de Atención Psicológica de la Universidad de Manizales en el período 2006-2010. La fuente de información consistió en las historias clínicas con base en las cuales realizaron un análisis cuantitativo. Por su parte, Barrera-Méndez, Bautista-Mercado y Trujillo-Martínez (2012), Hurtado-Betancur et al. (2011) y Quesada (2004) centraron sus investigaciones en la psicopatología de las personas, que acudieron a la consulta en diferentes centros de prácticas de estudiantes de Psicología, en México, Medellín y Buenos Aires, respectivamente.

Barrera-Méndez et al. (2012) construyeron una base de datos a partir de la información obtenida de la entrevista inicial. Hurtado-Betancur et al. (2011) indagaron, utilizando la entrevista estructurada conocida como Mini International Neuropsychiatric Interview, la prevalencia de trastornos mentales entre un grupo de personas voluntarias que consultaron en el servicio psicológico. Mientras que el interés de Quesada (2004) consistió en la construcción de un perfil psicopatológico de la población que fue atendida en la clínica psicológica, para lo cual utilizó la entrevista de admisión desde una aproximación psicoanalítica. En todos los casos, se utilizó un análisis cuantitativo de la información.

Además de las particularidades anteriormente señaladas, estos estudios se han realizado con objetivos tales como conocer mejor el perfil de las personas atendidas, evaluar el servicio brindado, identificar posibles líneas de investigación y estandarizar el lenguaje utilizado para el registro de los motivos de consulta (BarreraMéndez et al., 2012; Hurtado-Betancur et al., 2011; López-Jaramillo et al., 2012; Quesada, 2004). En el caso de la sistematización de la información del CAP de la UCR, la motivación es coincidente en algunos puntos con lo anteriormente señalado. El objetivo principal fue conocer las características sociodemográficas y el motivo de consulta de las personas que acudieron al CAP en búsqueda de atención psicológica.

Si bien los resultados de estos estudios no se pueden contrastar con los de la presente investigación, porque tienen objetivos, metodologías y aproximaciones diversas, sí permiten tener algunos parámetros de referencia para enriquecer la discusión de los resultados, aspecto que también es señalado por Quesada (2004) en su estudio. 
En esta gran diversidad, los estudios tienen como elementos comunes el hecho de que analizan un amplio número de casos (entre 103 y 1233 en los cuatro estudios reportados previamente), por lo cual el análisis que se realiza es cuantitativo. Asimismo, a la vez que refieren a la importancia de sistematizar la información, enfatizan en la complejidad de esta tarea, principalmente cuando se trata de la variable "motivo de consulta".

\section{Método}

Para cumplir los objetivos de la investigación se revisaron los expedientes de las personas atendidas en el CAP en el período 2004-2013. Se utilizó una aproximación cuantitativa con un alcance descriptivo (Hernández, Fernández \& Baptista, 2014) y se implementó la metodología de análisis de contenido. En esta metodología se utilizan categorías concretas para describir cuantitativamente textos de cualquier tipo (Crano, Brewer \& Lac, 2015; Newman, 2014). En este caso, los textos analizados fueron los expedientes de las personas atendidas.

\section{Población del estudio y fuentes de información}

Se tomó en cuenta el 100\% de los expedientes resguardados en el CAP correspondientes a los casos de atención clínica individual $(n=1045)$ de las prácticas profesionalizantes de los módulos y pasantías que se integraron a atender al CAP en el periodo comprendido entre los años 2004 y 2013. Por lo tanto, las fuentes de información fueron los expedientes. Las personas a quienes hacen referencia estos fueron en su mayoría mujeres (51.7\%), estudiantes (52.9\%), residentes de los cantones de San José (15.2\%), Montes de Oca (12.0\%), Goicoechea (10.8\%) y Desamparados (10.0\%) y con edades comprendidas entre los 1 y los 83 años $(M=25.46$ años, $D E=15.30, M d n=23$ años $)$.

\section{Criterios de inclusión y exclusión}

Para el análisis de los motivos de consulta, se tomó como criterio de inclusión que el expediente contara con el formulario de consentimiento informado firmado y que en él mismo la persona hubiera seleccionado la opción mediante la cual expresa que autoriza que la información de su expediente clínico sea utilizada para fines de investigación y académicos. En un total de 743 expedientes se contó con dicha autorización. De esta manera, es primordial aclarar que el análisis para las variables respecto al (a) expediente administrativo y a los (b) datos sociodemográficos se presenta sobre la población total (1045 expedientes), mientras que el análisis de los (c) motivos de consulta se realizó únicamente sobre los 743 expedientes de las personas que autorizaron la utilización de su expediente clínico, lo que representa un $71.1 \%$ del total.

\section{Instrumentos}

Como metodología de recolección de datos, se utilizó la técnica de análisis de contenido, la cual consiste en el análisis de textos a partir de categorías de las variables de interés, de manera que se llega a describir de forma cuantitativa textos originalmente cualitativos (Crano et al., 2015; Newman, 2014). En este caso, los textos analizados fueron los expedientes de las personas atendidas (boletas de ingreso, boletas de control de citas y consentimientos informados, etc.) y los informes finales de caso (los cuales son elaborados por el estudiantado que atendió a la persona). Para el registro de la información, se creó 
una base de datos electrónica donde cada expediente fue analizado y codificado con base en las variables que se describen a continuación:

1. Respecto al expediente administrativo: año (año en que se realizó el proceso clínico), tiempo de espera (número de días transcurridos desde el momento en que se solicitó la cita hasta el momento que se brindó la atención), número de sesiones (sesiones a las que asistió la persona), estudiante que atiende (nombre de la o el estudiante que atendió el caso), docente del curso (nombre de la o el docente que impartió el curso en el cual se inscribió la práctica clínica) y docente que supervisa el caso (nombre de la o el docente que supervisó directamente el caso).

2. Datos sociodemográficos de la persona atendida: sexo (sexo reportado por la persona: hombre, mujer u otro), edad (edad de la persona al momento de iniciar la atención), residencia (cantón y provincia reportado por la persona como su lugar de residencia) y ocupación (oficio de la persona al momento de la atención). Para la variable "ocupación" se utilizó, con leves modificaciones, la Clasificación de ocupaciones de Costa Rica (Instituto Nacional de Estadística y Censos, 2011). Se consignó la primera ocupación reportada en cada expediente y esta después se categorizó acorde a lo establecido por el INEC.

3. Motivo de consulta: las categorías de la variable "motivo de consulta" fueron construidas por el equipo de investigación. Las categorías construidas y presentadas en este artículo, aunque constituyen una síntesis, muestran la diversidad con la que se enunció la situación que hizo que las personas solicitaran atención psicológica. Es muy importante enfatizar que hay un criterio explícito, en el proceso de registro de los datos, de no adscribirse a las categorías de los manuales diagnósticos, ni tampoco pretender crear una nueva categorización con fines diagnósticos. Además, es fundamental mencionar que, del motivo de consulta inicial (aquel reportado por la persona en el momento que solicita el servicio), en la investigación se tomó en cuenta el motivo de consulta final (aquel elaborado a través del proceso terapéutico); sin embargo, por motivos de espacio, aquí solo se presentan los resultados elaborados con el motivo de consulta inicial. Las 20 categorías establecidas en este proceso son las siguientes:

a. Problemas familiares: Se trata de situaciones en que la persona reporta problemas referidos a lo familiar de una manera general.

b. Problemas de disciplina o crianza: Se refiere a las solicitudes de atención en las que la persona adulta expresa tener dificultades referidas a crianza o aquellas situaciones en que se indica que las personas menores de edad no están respondiendo a límites o no los han interiorizado.

c. Alteración emocional: Consiste en aquellas situaciones en que la persona manifiesta que sus emociones se encuentran alteradas. Las personas mencionaron aspectos tales como "nerviosismo", "manejo de emociones", "inseguridad", "falta de control" y "llanto", entre muchos otros.

d. Tristeza o depresión: Incluye situaciones en que la persona indica sentirse triste o deprimida. No refiere necesariamente a depresiones clínicamente identificadas.

e. Problemas de pareja: Alude a situaciones en que la persona identifica conflictos con su pareja (celos, violencia, convivencia, sexuales u otros). 
f. Separación o divorcio: Se vivencia mediante situaciones en que la persona está enfrentando una ruptura (divorcio/separación) en su relación de pareja.

g. Agresividad: Se refleja con situaciones en que la persona manifiesta dificultades para expresar su enojo, sea porque lo hace de manera agresiva (en algunos casos contra sí mismo) o porque no logra comunicarlo de manera asertiva.

h. Habilidades sociales: Son situaciones en que la persona alude a un problema vinculado a sus habilidades sociales (especialmente la carencia de las mismas) tales como la inseguridad al interactuar con otras personas.

i. Déficit atencional o hiperactividad: Se refiere a situaciones que son catalogadas como déficit atencional o hiperactividad por la persona que establece la solicitud. También, se incluyó cuando la persona indicó problemas de concentración.

j. Interés por un proceso terapéutico: Se refiere a situaciones en que la persona reporta que tiene interés en ser parte de un proceso terapéutico sin que exista un motivo particular de consulta; por ejemplo, estudiantes de Psicología que lo consideran como parte de su formación profesional, búsqueda de consejos, entre otras situaciones.

k. Proyecto de vida, carrera o trabajo: Se refiere a situaciones en que la persona consulta respecto a decisiones que debe tomar o la falta de claridad en lo relativo a su proyecto de vida, especialmente en el ámbito personal.

1. Problemas académicos: Se refiere a situaciones en que la persona consulta por dificultades vinculadas al ámbito académico, tales como bajo rendimiento y decisiones que debe tomar sobre su orientación vocacional.

m. Duelo: Se refiere a situaciones de duelo por diversas pérdidas, exceptuando las de pareja, que tienen una categoría aparte.

n. Abuso sexual o violación: Se refiere a sospechas de abuso sexual o violación en el presente o un abuso sexual o violación en el pasado y que la persona considera que sigue teniendo impacto en su vida.

o. Ansiedad o estrés: Se refiere a situaciones en que la persona reporta sentirse ansiosa o estresada, sea en términos generales o ante situaciones específicas.

p. Autoestima: Se refiere a problemas vinculados a la autoestima, usualmente se refiere "baja autoestima". También, se incluyó aquí cuando se hizo referencia a empoderamiento.

q. Problemas del desarrollo: Se manifiestan con situaciones en que se consulta porque se considera que la niña o el niño no tiene un desarrollo acorde con su edad.

r. Situación traumática: Vivencia de alguna situación traumática por parte de la persona; por ejemplo, encontrarse en medio de un desastre o una emergencia o por ser víctima de algún acto de delincuencia. 
s. Pensamientos que atormentan: Pensamientos recurrentes que le generan preocupaciones a una persona. Por ejemplo, "piensa mucho en problemas", "cargas y soluciones que no encuentra", entre otros.

t. Otros: Se refiere a las categorías con baja frecuencia, las cuales fueron unificadas bajo esta denominación.

Se consignó "No se indica" en aquellas situaciones en que no existió un registro en la boleta de ingreso ni en el expediente.

\section{Procedimientos}

Como ya se indicó, la información de los expedientes fue consignada en una base de datos, que contenía las variables de interés. Los datos fueron ingresados por cinco personas y todo el trabajo de digitación se llevó a cabo dentro del CAP, de manera que los expedientes no salieran de las instalaciones universitarias. La base de datos fue manejada en MS Excel (v. 15, para MacOS) e IBM SPSS Statistics (v. 20, para MacOS), programa con el cual se realizaron los análisis.

\section{Estrategia de análisis de datos}

Para cumplir con el objetivo general de la investigación, las estrategias estadísticas contemplaron análisis descriptivos a nivel de distribuciones de frecuencias, tablas cruzadas y medidas de tendencia central. En lo relativo a la categoría "motivos de consulta", en primer lugar, se consignó el motivo de consulta tal y como fue enunciado por las personas al tramitar la solicitud para ser atendidas. Posteriormente, mediante un análisis temático, se elaboró un listado de 53 categorías en las que se respetó el vocabulario de las personas consultantes y se conservaron, incluso, los motivos de consulta cuya frecuencia fue mínima al tabular los datos. Como estrategia complementaria, mediante el programa ATLAS.ti (v. 6, para Windows), se realizó un análisis de la frecuencia de aparición de palabras en el motivo de consulta exacto reportado por la persona atendida. Se verificó que los contenidos de las palabras más utilizadas en estos motivos de consulta estuvieran comprendidos en las categorías elaboradas en la etapa anterior. Asimismo, se contrastaron las categorías de motivos de consulta con las categorías utilizadas en otras investigaciones similares (Barrera-Méndez et al., 2012; López-Jaramillo et al., 2012; Quesada, 2004). Finalmente, las categorías afines fueron unificadas y aquellas con baja frecuencia se categorizaron bajo la etiqueta "Otros". Como resultado de este proceso, se contó con un listado final de 20 categorías de motivos de consulta.

\section{Resultados}

\section{Características generales}

\section{Cantidad de expedientes}

Para los diez años en estudio, se observó un aumento casi constante del total de expedientes registrados (tabla 1). Este aumento fue mayor a partir del 2009, de manera que en el 2013 se registró un total de 203 expedientes; es decir, un crecimiento de más de 1700\% en comparación con los casos registrado en el año de apertura. La mayor cantidad de personas atendidas registró haber participado de un solo 
proceso terapéutico en el CAP (96.6\%). Solamente 34 personas participaron en más de un proceso. En estos casos, resulta común haber llevado dos $(3.2 \%)$, pero muy poco frecuente haber llevado tres $(0.2 \%)$.

Tabla 1

Expedientes y estudiantes por año en el período 2004-2013

\begin{tabular}{|c|c|c|c|}
\hline \multirow[b]{2}{*}{ Año } & \multicolumn{2}{|c|}{ Expedientes } & \multirow{2}{*}{$\begin{array}{c}\text { Estudiantes } \\
n\end{array}$} \\
\hline & $n$ & $\%$ & \\
\hline 2004 & 11 & 1.1 & 10 \\
\hline 2005 & 50 & 4.8 & 36 \\
\hline 2006 & 29 & 2.8 & 21 \\
\hline 2007 & 79 & 7.6 & 50 \\
\hline 2008 & 70 & 6.7 & 44 \\
\hline 2009 & 113 & 10.8 & 80 \\
\hline 2010 & 142 & 13.6 & 85 \\
\hline 2011 & 163 & 15.6 & 92 \\
\hline 2012 & 162 & 15.5 & 98 \\
\hline 2013 & 203 & 19.4 & 103 \\
\hline
\end{tabular}

Nota: Cada estudiante puede haber atendido en más de un año.

\section{Cantidad de estudiantes $y$ docentes}

Al igual que con la cantidad de expedientes, la cantidad de estudiantes también aumentó con los años (tabla 1). Durante el año de apertura del CAP, se contabilizó la atención por parte de diez estudiantes, mientras que para el año 2013 la cantidad de estudiantes llegó a 103, lo que representa un aumento del 930\%. Lo común fue que las y los estudiantes atendieran uno (40.9\%) o dos (24.6\%) casos, aunque hubo algunas pocas personas que atendieron hasta ocho o nueve. En cuanto a docentes, se contabilizó un total de nueve docentes titulares de los cursos y de 31 docentes que supervisaron los casos a través de los diez años en estudio.

\section{Cantidad de sesiones}

De los 864 expedientes, en los que se logró determinar la cantidad de sesiones que implicó el proceso $(82.7 \%)$, se obtuvo una mediana de diez sesiones $(M=10.74$ sesiones, $D E=6.711)$. Hubo desde procesos clínicos en los que solo se realizó una sesión (situaciones en que las personas que consultan asisten únicamente a la primera sesión) hasta procesos con 77 sesiones. Sin embargo, en el 95\% de los casos la atención brindada tomó 22 sesiones o menos.

\section{Tiempo de espera}

De los 654 expedientes en los que se logró determinar el tiempo de espera entre la solicitud y el inicio del proceso $(62.6 \%)$, se obtuvo una mediana de 56.5 días $(M=97.59$ días, $D E=122.06)$. Se contó 
con casos que esperaron cero días hasta casos con 1060 días de espera. Sin embargo, en el 95\% de los casos la espera fue de 329 días (11 meses) de espera o menos.

Estos datos reflejan únicamente los casos de las personas que sí reciben la atención, porque existe un número de personas que solicita la atención y por motivos diversos no son atendidas; por ejemplo, porque no es posible localizarlas cuando se les asigna la cita o cuando se les contacta manifiestan que ya no tienen interés, entre otros.

\section{Características sociodemográficas}

Sexo

Respecto a la variable sexo, hubo una distribución balanceada. En aproximadamente la mitad de los casos, la persona atendida fue una mujer (51.7\%), mientras que en un $48.2 \%$, un hombre.

\section{Edad}

Las personas atendidas reportaron edades comprendidas entre los uno y 83 años de edad, con un promedio de 25.46 años $(D E=15.30)$. Los expedientes correspondieron en su mayoría a personas en la adultez joven (18 a 35 años, $n=419,40.1 \%$ ), en la infancia ( 1 a 12 años, $n=260,24.9 \%$ ) y en la adultez media ( 36 a 64 años, $n=257,24.6 \%$ ), aunque hubo expedientes de personas en la adolescencia (13 a 17 años, $n=81,7.8 \%$ ) y en la adultez mayor (65 a 83 años, $n=12,1.1 \%$ ).

En términos generales, la edad no difirió sustancialmente entre mujeres $(n=535, M=26.02, D E=$ $14.21)$ y hombres $(n=494, M=24.85, D E=16.39)$. Sin embargo, se observó una mayor proporción de infantes en el caso de los hombres (31.2\%) que en el caso de las mujeres (18.5\%), y una mayor proporción de personas en la adultez joven en el grupo femenino (50.2\%) que en el masculino (29.4\%).

\section{Provincia de residencia}

Costa Rica está organizada territorialmente en siete provincias: Alajuela, Cartago, Guanacaste, Heredia, Limón, Puntarenas y San José, las cuales se subdividen en 81 cantones. Respecto a la provincia de residencia, casi tres cuartas partes de los expedientes correspondieron a personas que reportaron vivir en la capital (71.8\%). Luego de San José, aquellas con mayor cantidad de personas atendidas fueron Cartago (14.4\%) y Heredia (7.6\%), pertenecientes al Área Metropolitana. Las restantes provincias, todas juntas, correspondieron a menos de un $4 \%$ de los expedientes atendidos (Alajuela $=3.2 \%$, Limón $=0.4 \%$, Puntarenas $=0.1 \%$ y Guanacaste $=0.1 \%$ ).

\section{Cantón de residencia}

Las personas atendidas en el CAP, durante estos diez años, residieron en un total de 45 cantones de todo el territorio nacional; es decir, poco más de la mitad de los cantones del país. Los mayores porcentajes de expedientes correspondieron a personas residentes en los cantones de San José (15.2\%), Montes de Oca (12.0\%), Goicoechea (10.8\%), Desamparados (10.0\%), Cartago (6.3\%) y La Unión (6.0\%).

\section{Ocupación}

Más de la mitad de los expedientes (52.9\%) correspondieron a personas cuya ocupación principal fue el estudio (tabla 2). Sin embargo, se atendió a personas con una amplia diversidad de ocupaciones, 
entre estas, trabajos profesionales (9.3\%), labores en servicios y comercios $(6.3 \%)$, amas de casa (6.3\%) y técnicos y profesionales de nivel medio (5.7\%). En cuanto al estudiantado, la mayoría contaba con entre seis y 12 años de edad (19.7\%) o entre 18 y 25 años de edad (15.7\%), por lo que se podría asumir que se trató principalmente de estudiantes de primaria y de educación superior.

Tabla 2

Expedientes por ocupación según sexo

\begin{tabular}{lccc}
\hline Ocupación & $\begin{array}{c}\text { Mujeres } \\
(n=540)\end{array}$ & $\begin{array}{c}\text { Hombres } \\
(n=504)\end{array}$ & $\begin{array}{c}\text { Total } \\
(n=1045)\end{array}$ \\
\hline Estudiantes & $52.2 \%$ & $53.8 \%$ & $52.9 \%$ \\
Menores a 6 años & $3.3 \%$ & $7.3 \%$ & $5.3 \%$ \\
De 6 a 12 años & $15.4 \%$ & $24.4 \%$ & $19.7 \%$ \\
De 13 a 17 años & $6.9 \%$ & $8.1 \%$ & $7.5 \%$ \\
De 18 a 25 años & $20.9 \%$ & $10.1 \%$ & $15.7 \%$ \\
De 26 a 61 años & $5.7 \%$ & $3.8 \%$ & $4.8 \%$ \\
Profesionales científicos e intelectuales & $8.9 \%$ & $9.7 \%$ & $9.3 \%$ \\
Trabajadores de los servicios y vendedores & $5.6 \%$ & $7.1 \%$ & $6.3 \%$ \\
Amas de casa & $12.2 \%$ & $0.0 \%$ & $6.3 \%$ \\
Técnicos y profesionales de nivel medio & $3.7 \%$ & $7.9 \%$ & $5.7 \%$ \\
Personal de apoyo administrativo & $4.3 \%$ & $2.4 \%$ & $3.3 \%$ \\
Ocupaciones elementales & $2.0 \%$ & $1.8 \%$ & $1.9 \%$ \\
Personas pensionadas & $1.3 \%$ & $2.0 \%$ & $1.6 \%$ \\
Oficiales, operarios y artesanos & $0.4 \%$ & $2.8 \%$ & $1.5 \%$ \\
Personas desempleadas & $0.9 \%$ & $1.8 \%$ & $1.3 \%$ \\
Operadores de instalaciones y máquinas & $0.0 \%$ & $1.6 \%$ & $0.8 \%$ \\
Agricultores, agropecuarios y pesqueros & $0.0 \%$ & $0.8 \%$ & $0.4 \%$ \\
Direcciones y gerencias & $0.0 \%$ & $0.4 \%$ & $0.2 \%$ \\
Categoría imprecisa & $3.9 \%$ & $4.4 \%$ & $4.1 \%$ \\
Sin respuesta & $4.6 \%$ & $3.6 \%$ & $4.2 \%$ \\
\hline
\end{tabular}

Se observaron algunas diferencias de ocupación según el sexo. Dentro de los expedientes de mujeres, hubo $12.2 \%$ correspondientes a la ocupación "ama de casa", mientras que esta categoría no se presentó en el caso de los hombres. Por el contrario, en la categoría de "técnicos y profesionales de nivel medio" los casos correspondientes a hombres (7.9\%) fueron más del doble de los de mujeres $(3.7 \%)$. En cuanto a los grupos etarios de estudiantes, aquellos en adultez joven fueron más del doble en el caso de 
las mujeres (20.9\%) que en el caso de los hombres (10.1\%), mientras que aquellos en la infancia tuvieron mayor presencia en el caso de los hombres (24.4\%) que en el grupo de mujeres (15.4\%).

\section{Motivos de consulta}

En la tabla 3 se pueden observar los principales motivos de consulta presentados en los expedientes revisados. En primer lugar, es importante resaltar que ninguna de las categorías de motivo de consulta aglutinó un alto porcentaje de los casos. Cada una de las 20 categorías construidas fue reportada en no más del 10\% de los expedientes analizados. Dentro de los motivos de consulta, que agruparon más de un 5\% de los casos, se encontraron categorías relacionadas con el ámbito de familia o pareja (problemas familiares $=10.0 \%$, problemas de disciplina o crianza $=7.8 \%$, problemas de pareja $=6.5 \%$, separación o divorcio $=5.8 \%$ ) y otras relacionadas con el componente emocional (alteración emocional $=7.4 \%$, tristeza o depresión $=7.3 \%$, agresividad $=5.1 \%$ ).

Tabla 3

Expedientes por motivo de consulta según sexo

\begin{tabular}{lccc}
\hline Motivo de consulta & $\begin{array}{c}\text { Mujeres } \\
(n=392)\end{array}$ & $\begin{array}{c}\text { Hombres } \\
(n=351)\end{array}$ & $\begin{array}{c}\text { Total } \\
(n=743)\end{array}$ \\
\hline Problemas familiares & $10.7 \%$ & $9.1 \%$ & $10.0 \%$ \\
Problemas de disciplina o crianza & $8.2 \%$ & $7.4 \%$ & $7.8 \%$ \\
Alteración emocional & $7.7 \%$ & $7.1 \%$ & $7.4 \%$ \\
Tristeza o depresión & $11.5 \%$ & $2.6 \%$ & $7.3 \%$ \\
Problemas de pareja & $5.4 \%$ & $7.7 \%$ & $6.5 \%$ \\
Separación o divorcio & $4.3 \%$ & $7.4 \%$ & $5.8 \%$ \\
Agresividad & $2.6 \%$ & $8.0 \%$ & $5.1 \%$ \\
Habilidades sociales & $3.3 \%$ & $4.0 \%$ & $3.6 \%$ \\
Déficit atencional o hiperactividad & $1.8 \%$ & $4.6 \%$ & $3.1 \%$ \\
Interés por un proceso terapéutico & $3.1 \%$ & $3.1 \%$ & $3.1 \%$ \\
Proyecto de vida, carrera o trabajo & $2.8 \%$ & $3.1 \%$ & $3.0 \%$ \\
Problemas académicos & $3.1 \%$ & $2.6 \%$ & $2.8 \%$ \\
Duelo & $4.1 \%$ & $1.1 \%$ & $2.7 \%$ \\
Abuso sexual o violación & $2.3 \%$ & $2.6 \%$ & $2.4 \%$ \\
Ansiedad o estrés & $3.1 \%$ & $1.7 \%$ & $2.4 \%$ \\
Autoestima & $2.8 \%$ & $1.7 \%$ & $2.3 \%$ \\
Problemas del desarrollo & $1.5 \%$ & $1.7 \%$ & $1.6 \%$ \\
Situación traumática & $2.3 \%$ & $0.3 \%$ & $1.3 \%$ \\
Pensamientos que atormentan & $2.0 \%$ & $0.3 \%$ & $1.2 \%$ \\
\hline
\end{tabular}

Continúa... 
Tabla 3

Expedientes por motivo de consulta según sexo

\begin{tabular}{lccc}
\hline Motivo de consulta & $\begin{array}{c}\text { Mujeres } \\
(n=392)\end{array}$ & $\begin{array}{c}\text { Hombres } \\
(n=351)\end{array}$ & $\begin{array}{c}\text { Total } \\
(n=743)\end{array}$ \\
\hline Otros & $9.7 \%$ & $5.1 \%$ & $7.5 \%$ \\
No se indica & $7.9 \%$ & $18.8 \%$ & $13.1 \%$ \\
\hline
\end{tabular}

\section{Motivos de consulta según sexo}

Al analizar estos motivos de consulta por sexo (tabla 3) se observaron varias diferencias. Un mayor porcentaje de mujeres reportó como motivo de consulta la tristeza o depresión $(11.5 \%$ versus 2.6 $\%$ ) y el duelo (4.1\% versus $1.1 \%$ ), mientras que un mayor porcentaje de hombres reportó como motivo de consulta la agresividad ( $8.0 \%$ versus $2.6 \%$ ) y déficit atencional o hiperactividad ( $4.6 \%$ versus $1.8 \%)$.

\section{Motivos de consulta según edad}

En la tabla 4 se puede observar la distribución de los expedientes por motivo de consulta según la edad de la persona atendida. En el caso de los infantes (1-12 años de edad), los motivos de consulta que aparecen con mayores porcentajes son problemas familiares $(14.4 \%)$, problemas de disciplina o crianza (13.9\%), agresividad (11.1\%), déficit atencional o hiperactividad (9.1\%), alteración emocional (7.2\%) y problemas académicos (7.2\%). Además, se observa, al establecer una comparación con los otros grupos etarios, que los motivos de consulta déficit atencional o hiperactividad (9.1\%), problemas académicos (7.2\%) y abuso sexual o violación $(5.3 \%)$, se presentan más frecuentemente en este grupo etario.

Tabla 4

Expedientes por motivo de consulta según edad

\begin{tabular}{lccccc}
\hline & \multicolumn{5}{c}{ Edad en años } \\
\cline { 2 - 6 } Motivo de consulta & $1-12$ & $13-17$ & $18-35$ & $36-64$ & $65-83$ \\
$(n=208)$ & $(n=54)$ & $(n=290)$ & $(n=181)$ & $(n=6)$ \\
\hline Problemas familiares & $14.4 \%$ & $14.8 \%$ & $9.0 \%$ & $5.5 \%$ & $0.0 \%$ \\
Problemas de disciplina o crianza & $13.9 \%$ & $16.7 \%$ & $3.8 \%$ & $5.0 \%$ & $0.0 \%$ \\
Alteración emocional & $7.2 \%$ & $5.6 \%$ & $7.2 \%$ & $8.3 \%$ & $16.7 \%$ \\
Tristeza o depresión & $1.9 \%$ & $7.4 \%$ & $9.7 \%$ & $8.3 \%$ & $50.0 \%$ \\
Problemas de pareja & $0.0 \%$ & $0.0 \%$ & $6.6 \%$ & $16.0 \%$ & $0.0 \%$ \\
Separación o divorcio & $2.9 \%$ & $0.0 \%$ & $5.9 \%$ & $11.0 \%$ & $0.0 \%$ \\
Agresividad & $11.1 \%$ & $7.4 \%$ & $2.1 \%$ & $2.8 \%$ & $0.0 \%$ \\
Habilidades sociales & $3.4 \%$ & $7.4 \%$ & $4.8 \%$ & $1.1 \%$ & $0.0 \%$ \\
& & & & & Continúa... \\
\hline
\end{tabular}


Tabla 4

Expedientes por motivo de consulta según edad

\begin{tabular}{|c|c|c|c|c|c|}
\hline \multirow[b]{2}{*}{ Motivo de consulta } & \multicolumn{5}{|c|}{ Edad en años } \\
\hline & $\begin{array}{c}1-12 \\
(n=208)\end{array}$ & $\begin{array}{c}13-17 \\
(n=54)\end{array}$ & $\begin{array}{c}18-35 \\
(n=290)\end{array}$ & $\begin{array}{c}36-64 \\
(n=181)\end{array}$ & $\begin{array}{c}65-83 \\
(n=6)\end{array}$ \\
\hline Proyecto de vida, carrera o trabajo & $0.0 \%$ & $0.0 \%$ & $5.5 \%$ & $3.3 \%$ & $0.0 \%$ \\
\hline Déficit atencional o hiperactividad & $9.1 \%$ & $3.7 \%$ & $0.3 \%$ & $0.6 \%$ & $0.0 \%$ \\
\hline Interés por un proceso terapéutico & $1.9 \%$ & $0.0 \%$ & $5.2 \%$ & $2.2 \%$ & $0.0 \%$ \\
\hline Problemas académicos & $7.2 \%$ & $3.7 \%$ & $1.0 \%$ & $0.6 \%$ & $0.0 \%$ \\
\hline Duelo & $1.4 \%$ & $3.7 \%$ & $3.1 \%$ & $2.2 \%$ & $33.3 \%$ \\
\hline Abuso sexual o violación & $5.3 \%$ & $1.9 \%$ & $2.1 \%$ & $0.0 \%$ & $0.0 \%$ \\
\hline Ansiedad o estrés & $0.0 \%$ & $1.9 \%$ & $4.1 \%$ & $2.8 \%$ & $0.0 \%$ \\
\hline Autoestima & $1.4 \%$ & $3.7 \%$ & $3.4 \%$ & $1.1 \%$ & $0.0 \%$ \\
\hline Problemas del desarrollo & $5.3 \%$ & $1.9 \%$ & $0.0 \%$ & $0.0 \%$ & $0.0 \%$ \\
\hline Situación traumática & $2.4 \%$ & $0.0 \%$ & $1.0 \%$ & $1.1 \%$ & $0.0 \%$ \\
\hline Pensamientos que atormentan & $0.0 \%$ & $0.0 \%$ & $2.1 \%$ & $1.7 \%$ & $0.0 \%$ \\
\hline Otros & $5.3 \%$ & $13.0 \%$ & $7.2 \%$ & $9.4 \%$ & $0.0 \%$ \\
\hline No se indica & $5.8 \%$ & $7.4 \%$ & $15.9 \%$ & $17.1 \%$ & $0.0 \%$ \\
\hline
\end{tabular}

Al analizar el grupo de adolescentes (13 a 17 años de edad), el motivo de consulta que los diferenció de los otros grupos se refirió a las habilidades sociales (7.4\%). Para este grupo etario, los motivos de consulta más frecuentes fueron los problemas de disciplina o crianza (16.7\%), problemas familiares $(14.8 \%)$, tristeza o depresión (7.4\%), agresividad (7.4\%) y habilidades sociales $(7.4 \%)$.

Respecto al grupo más numeroso, las personas adultas jóvenes (18 a 35 años de edad), estas se diferenciaron principalmente por el motivo de consulta relacionado con ansiedad o estrés $(4.1 \%)$, aunque sus principales motivos de consulta fueron tristeza o depresión (9.7\%), problemas familiares $(9.0 \%)$, alteración emocional $(7.2 \%)$, problemas de pareja $(6.6 \%)$, separación o divorcio $(5.9 \%)$ y problemas de disciplina o crianza $(3.8 \%)$.

Finalmente, en el caso de las personas en la adultez media (de 36 a 64 años de edad), los motivos en los que en mayor medida se diferenciaron de los restantes grupos fueron los problemas de pareja (16.0\%) y la separación o divorcio (11.0\%). Los principales motivos de consulta fueron los dos mencionados, además de tristeza o depresión (8.3\%) y alteración emocional (8.3\%).

En cuanto a las personas en la etapa de adultez mayor ( 65 a 83 años de edad), sus motivos de consulta fueron tristeza o depresión (50\%), duelo (33.3\%) o alteración emocional (16.7\%); sin embargo, los casos fueron pocos, por lo que no corresponde inferir diferencias con los restantes grupos etarios. 


\section{Motivos de consulta según sexo y edad}

En la tabla 5 se presentan los motivos de consulta segregados por sexo y edad. Las mujeres menores de edad no se diferenciaron particularmente de los otros grupos. Los principales motivos de consulta que enunciaron fueron problemas familiares (14.4\%), problemas de disciplina o crianza (13.5\%), alteración emocional (8.1 $\%$ ), problemas académicos (8.1\%), tristeza o depresión (6.3\%) y déficit atencional o hiperactividad (6.3\%).

\section{Tabla 5}

Expedientes por motivo de consulta según sexo y edad

\begin{tabular}{|c|c|c|c|c|}
\hline \multirow[b]{2}{*}{ Motivo de consulta } & \multicolumn{2}{|c|}{$\begin{array}{l}\text { Mujeres } \\
(n=391)\end{array}$} & \multicolumn{2}{|c|}{$\begin{array}{l}\text { Hombres } \\
(n=348)\end{array}$} \\
\hline & $\begin{array}{l}\text { Menores } \\
(n=111)\end{array}$ & $\begin{array}{l}\text { Mayores } \\
(n=280)\end{array}$ & $\begin{array}{l}\text { Menores } \\
(n=151)\end{array}$ & $\begin{array}{l}\text { Mayores } \\
(n=197)\end{array}$ \\
\hline Problemas familiares & $14.4 \%$ & $9.3 \%$ & $14.6 \%$ & $5.1 \%$ \\
\hline Problemas de disciplina o crianza & $13.5 \%$ & $6.1 \%$ & $15.2 \%$ & $1.5 \%$ \\
\hline Alteración emocional & $8.1 \%$ & $7.5 \%$ & $6.0 \%$ & $8.1 \%$ \\
\hline Tristeza o depresión & $6.3 \%$ & $13.6 \%$ & $0.7 \%$ & $4.1 \%$ \\
\hline Problemas de pareja & $0.0 \%$ & $7.5 \%$ & $0.0 \%$ & $13.7 \%$ \\
\hline Separación o divorcio & $1.8 \%$ & $5.4 \%$ & $2.6 \%$ & $11.2 \%$ \\
\hline Agresividad & $4.5 \%$ & $1.8 \%$ & $14.6 \%$ & $3.0 \%$ \\
\hline Habilidades sociales & $5.4 \%$ & $2.5 \%$ & $3.3 \%$ & $4.6 \%$ \\
\hline Déficit atencional o hiperactividad & $6.3 \%$ & $0.0 \%$ & $9.3 \%$ & $1.0 \%$ \\
\hline Interés por un proceso terapéutico & $2.7 \%$ & $3.2 \%$ & $0.7 \%$ & $5.1 \%$ \\
\hline Proyecto de vida, carrera o trabajo & $0.0 \%$ & $3.9 \%$ & $0.0 \%$ & $5.6 \%$ \\
\hline Problemas académicos & $8.1 \%$ & $1.1 \%$ & $5.3 \%$ & $0.5 \%$ \\
\hline Duelo & $2.7 \%$ & $4.6 \%$ & $1.3 \%$ & $1.0 \%$ \\
\hline Abuso sexual o violación & $4.5 \%$ & $1.4 \%$ & $4.6 \%$ & $1.0 \%$ \\
\hline Ansiedad o estrés & $0.9 \%$ & $3.9 \%$ & $0.0 \%$ & $3.0 \%$ \\
\hline Autoestima & $1.8 \%$ & $3.2 \%$ & $2.0 \%$ & $1.5 \%$ \\
\hline Problemas del desarrollo & $5.4 \%$ & $0.0 \%$ & $4.0 \%$ & $0.0 \%$ \\
\hline Situación traumática & $3.6 \%$ & $1.8 \%$ & $0.7 \%$ & $0.0 \%$ \\
\hline Pensamientos que atormentan & $0.0 \%$ & $2.9 \%$ & $0.0 \%$ & $0.5 \%$ \\
\hline Otros & $7.2 \%$ & $10.7 \%$ & $6.6 \%$ & $4.1 \%$ \\
\hline No se indica & $2.7 \%$ & $9.6 \%$ & $8.6 \%$ & $25.4 \%$ \\
\hline
\end{tabular}


Las mujeres mayores de edad se diferenciaron, principalmente, por los motivos de consulta de tristeza o depresión (13.6\%) y de "pensamientos que atormentan" (2.9\%). En este grupo, los principales motivos de consulta fueron tristeza o depresión (13.6\%), problemas familiares $(9.3 \%)$, alteración emocional $(7.5 \%)$, problemas de pareja $(7.5 \%)$ y problemas de disciplina o crianza $(6.1 \%)$.

En el caso de los hombres menores de edad, estos se diferenciaron de los restantes grupos principalmente por el motivo de consulta de agresividad (14.6\%). En este grupo, además del motivo mencionado, los motivos de consulta más frecuentes fueron problemas de disciplina o crianza (15.2\%), problemas familiares (14.6\%), déficit de atencional o hiperactividad (9.3\%) y alteración emocional $(6.0 \%)$.

Por último, el principal motivo que distinguió a los hombres mayores de edad fue el de separación o divorcio (11.2\%). Los motivos más frecuentes, además del anterior, fueron los problemas de pareja $(13.7 \%)$, alteración emocional $(8.1 \%)$, proyecto de vida, carrera o trabajo $(5.6 \%)$, problemas familiares $(5.1 \%)$ e interés por un proceso terapéutico $(5.1 \%)$.

\section{Discusión}

Como un primer aspecto, es necesario señalar que la investigación que toma como referencia los expedientes clínicos del CAP tiene una alta complejidad metodológica por varios aspectos tales como la diversidad de la información que está en cada uno de los expedientes y la lectura respectiva, según los diferentes paradigmas de la Psicología. Por lo tanto, la propuesta metodológica para continuar en esta línea de investigación debe irse construyendo tanto a partir del aporte de docentes y estudiantes que se vinculan con el CAP como de las experiencias de investigación que se realicen en el mismo. Respecto a los resultados expuestos, la discusión seguirá tres ejes: en primer lugar, algunas consideraciones sobre los aportes del CAP en términos del servicio brindado, un segundo eje en que se retoman las características sociodemográficas y, finalmente, lo relativo a los motivos de consulta.

Para los diez años en estudio, se observó un aumento constante del total de expedientes registrados. Se pasó de 11, en el 2004, a 203 en el 2013. Debe tenerse en cuenta que este aumento en el número de expedientes responde a varios factores que van desde la consolidación del proyecto a lo interno de la Escuela de Psicología hasta el aumento de los recursos con que se cuenta. Esto ha sido posible gracias a la designación de una persona coordinadora del proyecto, al aumento en el personal administrativo y el presupuesto asignado, así como un cambio en las instalaciones que redunda en la capacidad de atender un mayor número de personas cada día.

Lo anterior parece indicar que en el país hay una clara demanda de atención psicológica, pero que existen grandes barreras para brindarla, entre ellas las limitaciones de la oferta en el sector público, los altos costos económicos en el sector privado y el estigma que aún existe en amplios sectores de la población acerca de las implicaciones de la búsqueda de apoyo psicológico.

Si bien la UCR, como parte de su labor de Acción Social, provee este servicio, la discusión debe trascender tanto hacia el gremio profesional como hacia el Estado, respecto a la necesidad y la relevancia de facilitar el acceso de la población a profesionales en salud mental, tanto en procesos individuales como familiares y grupales, que apoyen a las personas a enfrentar sus momentos de crisis pero más allá de eso, a promover salud mental. 
En el caso de la UCR, proveer este servicio a través de espacios de inserción profesionalizante implica una importante responsabilidad ética y legal de parte del personal docente a cargo de los procesos formativos. Por ello, en estos casos la Escuela de Psicología, además de la o el docente titular del curso, otorga un nombramiento parcial para que otra u otro docente asuma labores en el proceso de supervisión. Esta razón resulta el motivo por el cual el número de docentes que supervisan es significativamente mayor que el número de docentes titulares de los cursos.

Un aspecto que se considera muy positivo es el tiempo de espera entre el momento en que se solicitó la atención y la primera cita en el CAP, porque los resultados ofrecen una mediana inferior a los dos meses. Este resultado es alentador tomando en cuenta que el CAP trabaja con recursos limitados y acorde con los periodos lectivos de la Universidad, por lo que en los periodos interciclo (diciembre-febrero y julio-agosto) se atienden muy pocos casos (usualmente de seguimiento de los procesos ya abiertos). Además, independientemente del momento del año en que llame la persona para solicitar la cita, la mayoría de los casos inician su atención en marzo o agosto.

En lo relativo al segundo eje de análisis referido a las características sociodemográficas, se observa que hubo una distribución similar por sexo. Existe una ligera diferencia a favor de las mujeres (51.7\% ante 48.2\%), similar a lo encontrado por Hurtado-Betancur et al. (2011). Por su parte, Quesada (2004) y López-Jaramillo et al. (2012) encontraron un porcentaje considerablemente mayor de mujeres que consultan. Estas autoras consideran que esto se explica porque las mujeres presentan menores prejuicios que los hombres al momento de buscar ayuda psicológica. Sin embargo, para poder entender la variable "sexo", es importante ver su relación con el rango etario.

El promedio de edad de las personas atendidas en el período del estudio fue de 25.46 años. En el caso de las mujeres, el grupo más grande se ubica entre los 18 y 25 años de edad, mientras que en el caso de los hombres se trata de niños menores de doce años de edad. Por lo tanto, se puede argumentar que las mujeres acuden a consulta por iniciativa propia, mientras que en el caso de los niños la consulta es motivada por personas adultas a cargo de ellos (habitualmente sus madres, docentes u otros familiares, por ejemplo). No obstante, este sería un tema para abordar en futuras investigaciones.

Respecto al lugar de residencia, la mayoría de las personas atendidas reportó los cantones de San José, Montes de Oca, Goicoechea y Desamparados, todos pertenecientes a la provincia de San José, lo cual puede explicarse por la cercanía geográfica de estos al CAP. Asimismo, en el caso de Montes de Oca debe tomarse en cuenta que en muchos casos se pudo identificar que la persona residía en ese cantón por razones de estudio, pero no se precisaba su lugar de origen.

Respecto a la variable ocupación, los servicios psicológicos fueron solicitados por personas de muy diversos sectores: desde amas de casa hasta profesionales, pasando por niveles técnicos y personas que laboran en el área de servicios. Existen algunas diferencias en las ocupaciones por sexo. Nuevamente, estos datos apuntan a la relevancia de habilitar opciones que sean económica y geográficamente accesibles a todos los sectores de la población.

No obstante, debemos llamar la atención sobre el hecho de que la mitad de las personas atendidas fueron estudiantes, principalmente de primaria y educación superior, según se puede deducir de sus edades. Hurtado-Betancur et al. (2011) y López-Jaramillo et al. (2012) también encontraron que el mayor 
número de personas consultantes fueron estudiantes de educación superior a nivel de pregrado. Esto podría explicarse por el hecho de que son centros adscritos a universidades, de tal manera que tanto el acceso a la información como a las instalaciones donde se brinda el servicio puede facilitarse para estudiantes de la institución. No obstante, López-Jaramillo et al. (2012) enfatizan que, pese a lo anterior, este es un ciclo de la vida en que las personas jóvenes se enfrentan a grandes desafíos. Es claro que la consulta no está motivada principalmente por problemas académicos o vocacionales, sino por diferentes situaciones en su ámbito familiar o en su vida personal. En todo caso, se evidencia que los servicios de salud mental se convierten en un recurso importante que la misma universidad debe ofrecer a sus estudiantes.

En síntesis, los datos muestran que a lo largo de diez años se ha atendido a personas muy diversas en cuanto a sus características sociodemográficas. Se muestra que no existen diferencias importantes respecto al sexo y edad de las personas, aunque sí tiende a haber una preponderancia de personas que residen en los cantones más cercanos a las instalaciones del CAP.

Como eje final, interesa retomar los resultados relativos a los motivos de consulta de las personas que fueron atendidas. En la revisión de estudios de otros países, en que se abordan los motivos de consulta como categoría de análisis, destaca un interés por uniformar criterios al tener como referencia el Manual Diagnóstico y Estadístico de los Trastornos Mentales (DSM-5, American Psychiatric Association, 2013) o la Clasificación Internacional de Enfermedades (CIE-10, World Health Organization, 2010). Además, el motivo de consulta inicial se establece con base en una entrevista de admisión o clínica (BarreraMéndez et al., 2012; Hurtado-Betancur et al., 2011; Quesada, 2004). En el caso de este estudio, el motivo de consulta inicial se construyó conforme a lo que la persona indicó al momento de solicitar la cita, porque se consideró valioso conocer la forma en la cual era enunciado aquello que motivaba la consulta, lo cual usualmente ocurre a través de una llamada telefónica. Al respecto, existe la limitación de que esta llamada suele implicar una indicación muy general, sea porque se sabe que está hablando con personal administrativo, o porque se tienen otras personas alrededor.

López-Jaramillo et al. (2012), si bien recomiendan utilizar un léxico basado en los manuales psiquiátricos para realizar diagnósticos más precisos que faciliten la atención y la investigación, reconocen que cuando existe una mayor correspondencia entre las necesidades de atención expresadas por las personas y el registro, se puede favorecer la permanencia de las personas consultantes en los procesos terapéuticos.

$\mathrm{Al}$ respecto, es muy importante resaltar que el CAP atiende a personas que consultan de manera libre y no necesariamente por referencia. Aunque en algunos casos se menciona que se solicita la atención por "referencia de la maestra", "referencia del médico", "referencia del Patronato Nacional de la Infancia", "referencia del Instituto Nacional de las Mujeres", estas no son referencias que se coordinan institucionalmente, sino que son recomendaciones que se plantean a las personas y son estas las que deciden si solicitan la atención o no.

Por lo tanto, más que atender criterios diagnósticos, lo que interesó en la investigación fue reconocer cuáles son los eventos que se convierten en desencadenantes para solicitar atención psicológica, así como el léxico que las personas utilizaron para hacerlo. Se observó una gran diversidad en la forma en que las personas se refieren a esto; en algunos casos, se utilizan categorías médicas-psiquiátricas como "depresión" o "déficit atencional"; en otros casos, se hace referencia a eventos como "divorcio" o "separación"; en otras ocasiones, se adjudica directamente a una persona. Se dice, por ejemplo, que "el niño es 
agresivo" y, en otras ocasiones, se señala una hipótesis de causalidad tal como "está triste por la muerte del abuelo". Este tema, sin duda, requerirá otra investigación que permita el seguimiento de este motivo de consulta inicial a través de un proceso clínico.

El motivo de consulta más común, entre hombres y mujeres, consiste en los problemas familiares. Sin embargo, llama la atención que en cuanto a emociones y sentimientos se observan diferencias acordes con los roles de género tradicionales. En el caso de las mujeres reportan en un mayor número sentimientos de tristeza y depresión, mientras que en el caso de los hombres predominan aquellos relacionados con la expresión y el manejo de la agresividad y la ira.

Asimismo, existen diferencias importantes entre los motivos de consulta cuando se considera la edad como una variable. Esto evidencia que las situaciones que se convierten en desencadenantes para solicitar atención psicológica están vinculadas a los procesos vitales. En el caso de niñas y niños menores de 12 años, así como el grupo de 13 a 17 años de edad, los motivos de consulta más comunes son problemas familiares, problemas de disciplina y crianza y agresividad. Sin embargo, debe llamarse la atención que estos motivos de consulta, a excepción del de problemas familiares, tienen el sesgo que son enunciados por las personas adultas (familiares, educadores/as, terapeuta). Sin establecer generalizaciones, hubo expedientes que registraron que la persona menor de edad no entendía por qué estaba en la consulta, pero seguía la directriz de la persona adulta que le acompañaba. Esta se identifica como una línea de investigación de interés como seguimiento al presente estudio. Barrera-Méndez et al. (2012) y LópezJaramillo et al. (2012) encontraron en sus estudios que los problemas de conducta son el primer motivo de consulta en niñas y niños, y el segundo cuando se consideran todas las personas menores de edad. Todo esto permite plantear la hipótesis de que los motivos de consulta de niñas y niños están referidos al ámbito doméstico, pero se tienden a enfocar en la conducta del niño o la niña para ser llevados a consulta.

En el grupo de 18 a 35 años, entendido acorde con la Ley General de la Persona Joven (Asamblea Legislativa, 2002) como personas adultas jóvenes, los principales motivos de consulta son la tristeza, la depresión, los problemas familiares y la alteración emocional. Este es un período de la vida en el que usualmente las personas están construyendo y definiendo su proyecto de vida (vocacional, laboral, de pareja, etc.). Sin embargo, se están enfrentando a cambios y rupturas; es decir, las personas encuentran grandes diferencias respecto a su realidad y el ideal buscado, las cuales se manifiestan en los malestares señalados como motivos de consulta.

Por su parte, en el grupo de 36 a 64 años de edad, se evidencia cómo el motivo de consulta inicial se traslada del ámbito familiar al de pareja, porque los problemas de pareja aparecen como el primer motivo de consulta, seguido de separación o divorcio. Estos resultados son coincidentes con los datos de Barrera-Méndez et al. (2012) y López-Jaramillo et al. (2012), quienes también encontraron aspectos relativos a las relaciones de pareja como uno de los principales motivos entre la población adulta, que fue atendida en sus centros de atención psicológica.

Por último, para el grupo de personas adultas mayores, no se pueden establecer generalizaciones porque únicamente hay seis casos registrados. Sin embargo, se puede observar que los motivos de consultas estuvieron centrados en tres únicas razones: tristeza y depresión, duelo y alteración emocional. Estos 
motivos no resultan extraños para las condiciones de vida de la población adulta mayor en nuestro país, así como por ser una etapa de la vida en que las pérdidas por diversas razones se vuelven más frecuentes.

En síntesis, respecto a los motivos de consulta, existe una amplia diversidad de circunstancias que motivan la búsqueda de atención psicológica y estas refieren a situaciones complejas por las que están pasando las personas y los grupos familiares, al menos, de la población del área metropolitana, que está acudiendo a solicitar atención en el CAP. Tal y como fue planteado desde sus objetivos, el estudio en que se basa este artículo es descriptivo y general, pero se espera que futuras investigaciones puedan profundizar sobre temáticas o poblaciones específicas. Los porcentajes por motivos de consulta son bajos, lo cual daría fundamento para realizar estudios de corte cualitativo centrados en problemáticas específicas.

Para finalizar, la construcción de las categorías para sistematizar los motivos de consulta fue uno de los mayores retos de esta investigación. En este proceso reflexivo, quedó claro que las decisiones tomadas implicaron tomar posición sobre las temáticas planteadas. Augsburger (2004) lo señala claramente:

Las clasificaciones son resultado de los modos como se percibe, se distingue y se organiza un sector determinado de la realidad. Se trata de un trabajo de abstracción de los fenómenos que se presentan siendo éstos sistemáticamente distribuidos por el pensamiento según algunas de sus propiedades o atributos, operando al mismo tiempo la existencia de esa clasificación como organizadora del pensamiento y de la visión que se tiene sobre la realidad. Es decir que la forma como se clasifican los eventos tiene una implicación fundamental sobre el modo como se representan y se interviene sobre ellos (p. 79).

La autora citada afirma que si bien ha habido avances teóricos sobre la comprensión del rol que la cultura y la organización social juegan tanto en la producción de la salud mental como de la enfermedad mental, el avance desde las metodologías para su estudio (y particularmente la epidemiología) se ha quedado rezagado en el marco de categorías diagnósticas que ineludiblemente remiten a la enfermedad mental. Por lo tanto, la propuesta de esta investigación de trabajar en la construcción de categorías propias no pretende de ninguna manera dialogar con los manuales diagnósticos o los enfoques de trabajo que los adhieren, sino iniciar un trabajo desde "abajo" que, en caso de tener seguimiento como línea de investigación por parte de la Escuela de Psicología, podría ir avanzando hacia algún sistema de categorías que pueda trabajarse desde la diversidad paradigmática; o bien, que cada paradigma elabore el sistema propio, pero siempre teniendo como objetivo común la expresión del malestar y del sufrimiento humano y no la categorización patológica.

Para concluir, es importante mencionar que durante el proceso de investigación se presentaron algunas dificultades metodológicas que, más allá de su resolución en el proceso, se constituyen en un resultado que aporta información muy valiosa para el trabajo en el CAP y para la Escuela de Psicología. En un inicio, por la organización de los expedientes, no se podía conocer la cantidad de documentos disponibles y esto dificultó la organización del trabajo para garantizar el cumplimiento de los plazos previstos. A partir de esta experiencia, se considera necesario generar e implementar lineamientos institucionales tanto en el archivo como en el resguardo de los expedientes. En segundo lugar, otra dificultad fue la desorganización de la información a lo interno de cada expediente, lo cual hizo más lento el proceso de revisión.

En el proceso de registro de los datos y de construcción de la matriz, se siguieron las coordenadas de los procesos de atención individual. En un inicio, los expedientes de atención de pareja o familia se fueron 
identificando como pendientes porque su registro no se ajustaba a los parámetros establecidos. Como resultado de varias reuniones del equipo en que se abordó este tema, se decidió excluir estos expedientes de la investigación, porque el registro implica una lógica diferente que vendría a afectar el análisis de los datos. Por ejemplo, no siempre había información personal y demográfica precisa de todas las personas atendidas, porque el expediente identificaba al conjunto de las personas atendidas y no a cada una de ellas; por ejemplo, en el expediente consta "familia (apellido)" [Familia Rodríguez]. Por lo tanto, se identifica tanto como una limitación del estudio como un reto para abordar a futuro, porque no es una variable que se debe incorporar solo desde lo logístico, sino también desde el modelo de abordaje clínico de estos procesos.

En otro orden de cosas referidas al abordaje clínico, hubo grandes limitaciones encontradas en la "construcción" del caso que el estudiantado presenta en sus informes. Fue usual que dichos documentos presentasen el caso en términos muy descriptivos; es decir, un relato de lo dicho por la o el paciente en las sesiones, pero sin una elaboración clínica del mismo. De tal manera, que el registro de esta categoría implicó un gran desafío.

A partir de lo expuesto anteriormente y teniendo como referente el proceso del trabajo realizado (no los resultados en sí mismos), la investigación que toma como referencia los expedientes clínicos del CAP tiene una alta complejidad metodológica, la cual debe irse construyendo tanto a partir del aporte de docentes y estudiantes que se vinculan con el CAP como con las experiencias de investigación que se realicen.

Finalmente, el alcance de este estudio es totalmente descriptivo y su meta fue justamente el registro y la sistematización de la información. Sin embargo, se espera que la base de datos pueda ser utilizada como referente en futuros proyectos de investigación que tengan interés en efectuar indagaciones más específicas y de orden cualitativo respecto a poblaciones o temáticas de interés de la persona investigadora. Por ejemplo, tomar alguna de las categorías construidas y que sea un tema de relevancia nacional (abuso sexual y violación, solo por citar un ejemplo) y hacer una revisión de estos expedientes para identificar particularidades del proceso psicoterapéutico o tener mayor claridad sobre el perfil de las personas que consultan por ese motivo. Otra línea de interés es estudiar la relación que existe entre el motivo de consulta que enuncia la persona al solicitar la cita y el motivo de consulta final que es identificado por el o la terapeuta como resultado del proceso. En último lugar, al estudiar los motivos de consulta, se observaron diferencias acordes con los roles de género. En el caso de las mujeres, reconocen y expresan sentimientos de tristeza, depresión y ansiedad, mientras que en el caso de los hombres los motivos de consulta tienen que ver con la expresión y el manejo de la agresividad y la ira. Por lo tanto, la variable de género se perfila como de interés para plantear futuras investigaciones.

\section{Referencias}

American Psychiatric Association. (2013). Diagnostic and statistical manual of mental disorders (5a. ed.). Virginia, Estados Unidos: Autor.

Asamblea Legislativa de la República de Costa Rica. (2002). Ley general de la persona joven (No. 8261). San José, Costa Rica: Autor.

Augsburger, A. C. (2004). La inclusión del sufrimiento psíquico: Un desafío para la epidemiología. Psicologia \& Sociedade, 16(2), 71-80. 
Barrera-Méndez, L. P., Bautista-Mercado, E. \& Trujillo-Martínez, A. (2012). Prevalencia de problemas psicológicos detectados en un centro de educación y desarrollo humano. Enseñanza e Investigación en Psicología, 17(1), 13-27.

Crano, W. D., Brewer, M. B. \& Lac, A. (2015). Principles and methods of social research (3a. ed.). Nueva York, Estados Unidos: Routledge.

Hernández, R., Fernández, C. \& Baptista, M. (2014). Metodología de la investigación (6a. ed.). México: McGraw-Hill.

Hurtado-Betancur, H., Sánchez-Peláez, V., Bedoya-Gómez, L., Londoño, N., Juárez, F., Marín, C. ... Gómez, Y. (2011). Prevalencia de trastornos mentales en usuarios de consulta psicológica en una universidad colombiana. Revista de Psicología de la Universidad de Antioquia, 3(1), 59-71.

Instituto Nacional de Estadística y Censos (2011). Clasificación de ocupaciones de Costa Rica (COCR-2011). Recuperado de http://www.inec.go.cr

López-Jaramillo, M. R., Montoya-Londoño, D. M. \& Dussán-Lubert, C. (2012). Caracterización de los asistentes al centro de atención psicológica de la Universidad de Manizales, 2006-2010. Hacia la Promoción de la Salud, 17(2), 149-166.

Molina, L. (2015). Centro de Atención Psicológica y Proyección Comunitaria de la Escuela de Psicología: Información académica del proyecto. Recuperado de https://www.bite.ucr.ac.cr/bite/ pdf info_academica by codigo activo/ED-2157

Newman, W. L. (2014). Social research methods: Qualitative and quantitative approaches (7a. ed.). Essex: Pearson.

Quesada, S. (2004). Estudio sobre los motivos de consulta psicológica en una población universitaria. Universitas Psychologica, 3(1), 7-16.

Salazar, Z. (2009). Caracterización de la Escuela de Psicología Universidad de Costa Rica: Experiencia de la autoevaluación y la reacreditación. San José, Costa Rica: Escuela de Psicología de la Universidad de Costa Rica.

World Health Organization (2010). International statistical classification of diseases and related health problems, (10th revision). Recuperado de http://apps.who.int/classifications/icd10/browse/2010/en

Recibido: 21 de abril de 2016 Revisión recibida: 28 de marzo de 2017

Aceptado: 18 de mayo de 2017 


\section{Sobre las autoras y el autor:}

Adriana Maroto Vargas es Máster en Sociología y Licenciada en Psicología. Ambos títulos otorgados por la Universidad de Costa Rica, institución en la cual labora como docente e investigadora. Entre sus temas de interés se encuentran el feminismo, los derechos reproductivos, la sociología de la religión y la salud comunitaria. Cuenta con publicaciones académicas y ha participado en conferencias y congresos nacionales e internacionales.

Lucía Molina Fallas es psicoanalista, Magíster en Teoría Psicoanalítica, profesora de la Universidad de Costa Rica (Costa Rica) y coordinadora del Centro de Atención Psicológica, de la Escuela de Psicología de esa misma casa de estudios. Forma parte de la Asociación Costarricense de Investigación y Estudio del Psicoanálisis y del Comité Editorial de la Revista Otra Escena. Cuenta con publicaciones y participaciones en congresos nacionales e internacionales. Atiende consulta privada.

Jorge Esteban Prado-Calderón es Licenciado en Psicología y estudiante de la Maestría en Ciencias Cognoscitivas, ambas de la Universidad de Costa Rica (UCR). Se desempeña como docente e investigador en la Escuela de Psicología y en la Sede de Guanacaste de la UCR, donde imparte cursos de investigación, historia de la psicología, y psicología cognitiva. Cuenta con 16 publicaciones académicas, 15 conferencias, diez proyectos de investigación y ha participado en comités científicos de simposios y congresos nacionales e internacionales.

Publicado en línea: 26 de junio de 2017 